\section{BMJ Open Respiratory Research}

\title{
Management of tracheostomies in the intensive care unit: a scoping review
}

\author{
Kirsty A Whitmore (D) , ${ }^{1,2}$ Shane C Townsend, ${ }^{1}$ Kevin B Laupland (D) ${ }^{1,3}$
}

To cite: Whitmore KA, Townsend SC, Laupland KB. Management of tracheostomies in the intensive care unit: a scoping review. BMJ Open Resp Res 2020;7:e000651. doi:10.1136/ bmjresp-2020-000651

- Additional material is published online only. To view please visit the journal online (http://dx.doi.org/10.1136/ bmjresp-2020-000651)

Received 26 May 2020 Revised 20 June 2020 Accepted 25 June 2020

\section{Check for updates}

(C) Author(s) (or their employer(s)) 2020. Re-use permitted under CC BY-NC. No commercial re-use. See rights and permissions. Published by BMJ.

${ }^{1}$ Department of Intensive Care Services, Royal Brisbane and Women's Hospital, Herston, Queensland, Australia ${ }^{2}$ Faculty of Medicine, The University of Queensland, Saint Lucia, Queensland, Australia

${ }^{3}$ Faculty of Health, Queensland University of Technology, Brisbane, Queensland,

Australia

Correspondence to Professor Kevin B Laupland; Kevin.Laupland@qut.edu.au

\section{ABSTRACT}

Objectives While there is an extensive body of literature surrounding the decision to insert, and methods for inserting, a tracheostomy, the optimal management of tracheostomies within the intensive care unit (ICU) from after insertion until ICU discharge is not well understood. The objective was to identify and map the key concepts relating to, and identify research priorities for, postinsertion management of adult patients with tracheostomies in the ICU.

Design Scoping review of the literature.

Data sources PubMed, Embase and Cumulative Index to Nursing and Allied Health Literature were searched from inception to 3 October 2019. Additional sources were searched for published and unpublished literature. Eligibility criteria We included studies of any methodology that addressed the a priori key questions relating to tracheostomy management in the ICU. No restrictions were placed on language or year of publication. Data extraction and synthesis Titles and abstracts were screened by two reviewers. Studies that met inclusion criteria were reviewed in full by two reviewers, with discrepancies resolved by a third. Data were extracted for included studies, and results mapped along the prespecified research questions.

Results 6132 articles were screened, and 102 articles were included for detailed analysis. Protocolised weaning was found to be successful in liberating patients from the ventilator in several cohort studies. Observational studies showed that strategies that use T-pieces and high-flow oxygen delivery improve weaning success. Several lines of evidence, including one clinical trial, support early cuff deflation as a safe and effective strategy as it results in a reduced time to wean, shorter ICU stays and fewer complications. Early tracheostomy downsizing and/or switching to cuffless tubes was found to be of benefit in one study. A substantial body of evidence supports the use of speaking valves to facilitate communication. While this does not influence time to wean or incidence of complications, it is associated with a major benefit in patient satisfaction and experience. Use of care bundles and multidisciplinary team approaches have been associated with reduced complications and improved outcomes in several observational studies.

Conclusions The limited body of evidence supports use of weaning protocols, early cuff deflation, use of speaking valves and multidisciplinary approaches. Clinical trials examining post-tracheostomy management strategies in ICUs are a priority.

Tracheostomy is performed in approximately $10 \%-15 \%$ of patients who are admitted to intensive care units (ICU). ${ }^{1}$ This procedure,

\section{Key messages}

What is the key question?

What evidence exists regarding postinsertion tracheostomy management in the ICU, and where are the gaps in the evidence?

What is the bottom line?

- There is a lack of quality research on this topic, with significant variations in practice primarily driven by clinician experience and local preferences.

\section{Why read on?}

- This review scopes and summarises all available literature on this topic and identifies priority areas for future studies regarding tracheostomy management.

which involves either percutaneous or surgical placement of a tube across the anterior neck into the airway, may be indicated for relief of airway obstruction, facilitation of pulmonary toilet and for facilitation of wean from mechanical ventilation. ${ }^{2}$ In addition, tracheostomy may allow a reduction of sedation, improve patient safety and comfort, and reduce overall costs of care. ${ }^{34}$ There is a vast body of literature published on the indications, timing and technique of tracheostomy insertion, including several meta-analyses. ${ }^{5-9}$ Likewise, there are a number of systematic reviews, surveys and expert guidelines on the timing and act of decannulation, or removal of the tracheostomy. ${ }^{1011}$

While there is extensive research on tracheostomy insertion and decannulation, their optimal management in ICUs between the time of insertion and ICU discharge has received little attention. Management aspects such as optimal cuff management, tracheostomy changes or downsizing, and weaning approaches are typically directed by individual expertise, anecdotal experience and local preferences.

The objective of this study was to conduct a scoping review to systematically explore and map the key concepts and gaps in the literature, in order to identify research priorities relating to the management of intensive care 
patients with tracheostomies from after insertion of the tracheostomy to the time of ICU discharge. Scoping reviews are used to determine the scope or coverage of a body of literature on an emerging topic and aims to identify and map the available evidence to provide an overview that may guide further research. ${ }^{12}{ }^{13}$ While a systematic review can answer a single precise question to inform clinical practice, a scoping review addresses the extent, range and nature of the evidence, and identifies common concepts or gaps. Given the limited volume and heterogeneous nature of the literature published on this topic, the scoping review methodology was most suited to the objectives of this review. It is our hope that this review may subsequently guide future high-level research, such as clinical trials and systematic reviews to answer more precise questions and address research priorities that have been identified.

\section{METHODS}

The study protocol was established a priori and was developed as outlined by the members of the Joanna Briggs Institute and members of the Joanna Briggs Collaborating Centres. ${ }^{12}$ The specific research questions were, 'What evidence exists regarding the ideal strategies to:

1. liberate patients from the ventilator?

2. manage cuff inflation and deflation?

3. manage tracheostomy change and revision?

4. optimise speech and communication?

5. optimise swallowing and oral intake?'

\section{Eligibility criteria}

Studies of any methodology that addressed one or more of the research questions were considered. Clinical studies were selected for inclusion if:

1. the study subjects were adults (age $\geq 18$ years) admitted to medical, general surgical or specialty surgical ICUs;

2. a tracheostomy was inserted during the current hospital admission;

3. the study related to a period after insertion of the tracheostomy and prior to ICU discharge; and

4. the subjects had respiratory failure requiring mechanical ventilation.

Studies that met all inclusion criteria but did not address one of the prespecified research questions were included. This approach was taken to enable this review to address concepts that emerged during the review and had not previously been considered.

Studies that focused on patients who had chronic tracheostomies or who had a tracheostomy placed prior to ICU admission for reasons other than for acute respiratory failure (eg, elective upper airway surgery) were excluded. Studies that focused on the indications for, timing of, and performance of the tracheostomy insertion procedure were excluded. Studies that did not involve patients as subjects (eg, studies relating to medical devices not tested in a live patient population) were only included if they addressed research questions relevant to the patient population in the inclusion criteria. Review articles and editorials that did not contain novel information were excluded.

\section{Search methodology}

An initial electronic search was conducted of three key databases (PubMed, Embase and Cumulative Index to Nursing and Allied Health Literature) from inception through to 3 October 2019. The keywords used were "ICU" or "intensive care unit" and "tracheostomy". The study strategy is available in as an electronic supplement (e-supplementary file 1) The search was not limited by design, language or year of study. The reference lists of included articles, review articles and editorials were searched for additional potential studies.

Titles and abstracts were screened independently by two authors (KAW and KBL) for potential inclusion. Articles that met inclusion criteria based on title and abstract review were included for full text review. Fulltext articles were retrieved and reviewed independently by two authors (KAW and SCT) to assess whether they met the inclusion criteria. Discrepancies between the two reviewers were resolved by a third. Following the compilation of a list of articles for inclusion, data were extracted by one of the authors (KAW), discussed and reviewed by all authors, and results mapped along the prespecified research questions. Data extracted included first author's surname, year of publication, setting, country of origin, aims or objectives, study population and size (if applicable), study design, duration of intervention (if applicable) and key findings. Analysis was descriptive.

\section{Patient and public involvement}

This review includes studies that consider the patient experience of tracheostomy management in the intensive care, including qualitative or phenomenological studies. Patients and the public were not involved in the completion of this review.

\section{RESULTS}

Electronic searches conducted retrieved 8801 citations across the PubMed (2198), Embase (5805) and Cumulative Index to Nursing and Allied Health Literature (798) databases. An additional 47 citations were identified from other sources, including from hand-searched reference lists, guidelines and resources published by professional bodies, online searches and conference abstracts. Following the removal of duplicates and the application of study inclusion and exclusion criteria, 102 articles were included in the final review, as detailed in figure 1.

Of the 102 articles included, 25 were only available as abstracts published in conference proceedings. Given that the objective of this review was to map all available evidence, these abstracts were included. Care should be taken in the interpretation of such results; therefore, abstract-only publications have been noted as such 


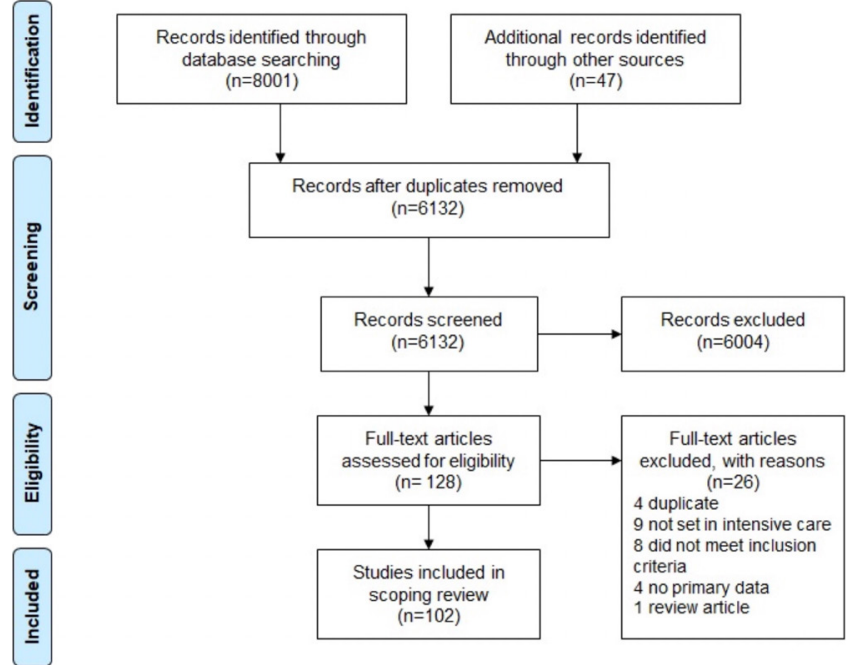

Figure 1 Preferred Reporting Items for Systematic Reviews and Meta-Analyses flow diagram for study identification and inclusion.

throughout this review. Articles included in this review were published between 1987 and 2019, with the majority published since 2013, as shown in figure 2. Studies were conducted in 21 different countries, with the most common being the USA $(n=22)$, the UK $(n=16)$ and Australia $(\mathrm{n}=15)$. The remaining 48 studies originated from Europe $(n=31)$, Asia $(n=7)$, South America $(n=5)$, Africa $(n=2)$ and North America $(n=1)$. The country of origin was not specified in three cases. Most $(n=69)$ of the identified articles addressed one or more of the five research questions, as mapped in figure 3. Studies that addressed more than one key question were categorised based on the question that was most extensively addressed. Thirty-three articles addressed other topics and have been included in a separate 'Other' category. The results of the studies are summarised in the electronic supplement e-supplementary file 2 . The key findings, categorised by theme, have been summarised in table 1 .

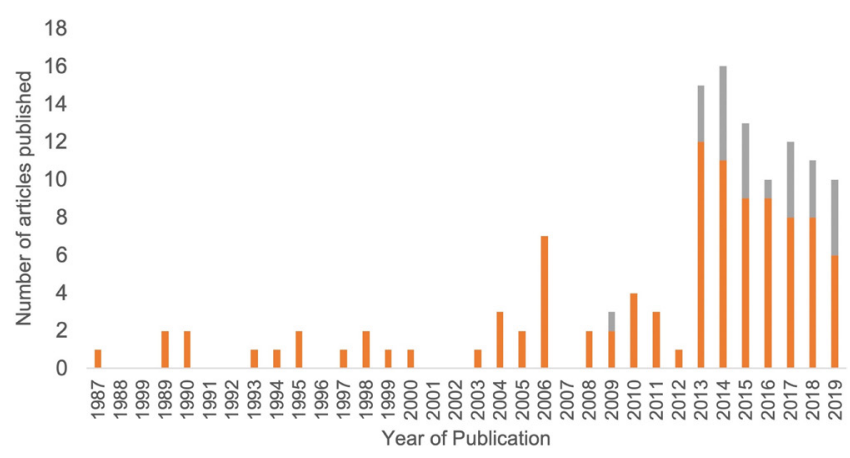

Figure 2 Annual publications investigating postinsertion tracheostomy management strategies for patients admitted to intensive care units.

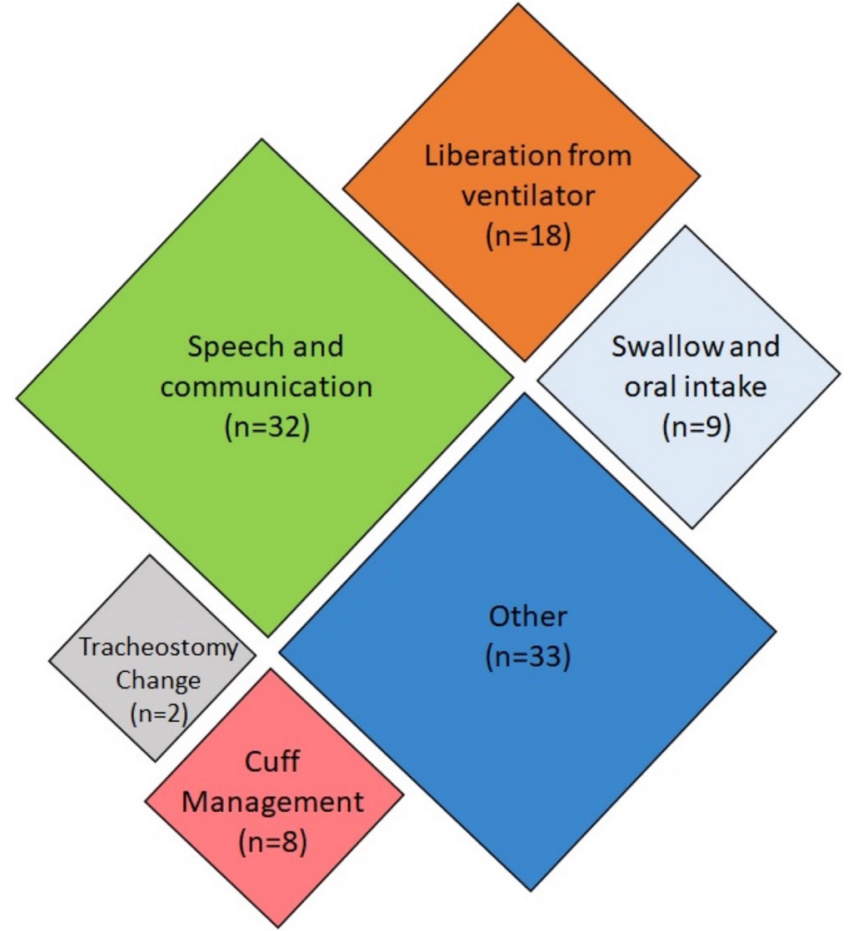

Figure 3 Mapping of included articles based on key research question addressed.

\section{Liberation from ventilator}

Twenty studies investigated topics related to weaning from mechanical ventilation. Five studies compared protocolised weaning models and reported successful weaning in $77 \%-89 \%$ of patients. Those studies reported that the use of protocols resulted in no difference in the time to weaning, length of stay, rates of emergent reintubation or readmission to the ICU. ${ }^{14-18}$ Two studies addressed nurse-led and protocolised weaning. One randomised trial compared nurse-led protocolised weaning and physician-led weaning, and found that patients were more likely to be weaned prior to ICU discharge in the nurse-led group (77\% protocolised vs $45 \%$ physician led, $\mathrm{p}=0.031) .{ }^{1416}$ A prospective study involving 192 patients found that there was no difference in weaning outcomes when medical management was provided by an attending physician and either a unit-based nurse practitioner, or a critical care rotational fellow. ${ }^{16}$

Three studies evaluated predictors of successful weaning outcomes or decannulation. Two studies found that weaning or decannulation success was more likely in patients that were able to increase the force-generating capacity of the diaphragm. ${ }^{19}{ }^{20}$ An observational study of 49 patients found that the ability to generate peak cough flows of greater than $160 \mathrm{~L} / \mathrm{min}$ was predictive of successful decannulation. ${ }^{19}$ In a retrospective audit of 129 patients in a Dutch medical-surgical ICU, patients whose primary pathology was neurosurgical or cardiopulmonary were statistically more likely to have shorter weaning times (3-7 days) when compared with medical (9 days) or surgical ( 8 days) patients. ${ }^{17}$ 


\begin{tabular}{ll}
\hline Table 1 Key findings & \\
\hline Liberation from ventilation & $\begin{array}{l}\text { Protocolised weaning and high-flow oxygen may improve weaning outcomes. } \\
\text { Automatic tube compensation mode may reduce additional work of breathing, } \\
\text { compared with other modes. } \\
\text { Early cuff deflation promotes vocalisation and swallowing, and may reduce length of } \\
\text { stay, time to decannulation and risk of nosocomial pneumonia. }\end{array}$ \\
\hline Tracheostomy change and revision & $\begin{array}{l}\text { Early tube changes may be associated with earlier use of speaking valves, oral intake } \\
\text { and shorter ICU stays. }\end{array}$ \\
\hline $\begin{array}{l}\text { Optimisation of speech and } \\
\text { communication }\end{array}$ & $\begin{array}{l}\text { In-line speaking valves may improve gas distribution and alveolar recruitment. Speech } \\
\text { and communication significantly impact on patient quality of life. }\end{array}$ \\
\hline Optimisation of swallow & $\begin{array}{l}\text { Swallowing physiology can be adversely affected by high cuff pressures. Despite most } \\
\text { patients commencing oral intake with tracheostomies in situ, only } 40 \% \text { commence oral } \\
\text { intake while in ICU. }\end{array}$ \\
\hline
\end{tabular}

ICU, intensive care unit.

An observational study of 25 patients compared T-piece ventilation and continuous positive pressure ventilation during weaning and found that T-piece ventilation resulted in improved arterial and central venous oxygenation. ${ }^{21}$ One letter reported that configuring the mixing tube between the T-piece and Venturi mask connector improved oxygenation. ${ }^{22}$ Three articles, including a case report and an abstract, discussed the use of highflow oxygen during weaning. ${ }^{23-25}$ One of these studies, a randomised cross-over trial, found that during cuff deflation, the use of high-flow oxygen improved oxygenation when compared with T-piece ventilation alone $(\mathrm{p}<0.02) .^{23}$ Two studies evaluated different types of humidification systems and concluded that heat-moisture-exchange systems and heated humidifiers provided adequate humidification, and that cold humidifiers provided inadequate humidification in $50 \%$ of patients. ${ }^{26} 27$

Two studies addressed aspects of respiratory physiology during weaning. A clinical trial of 10 patients found that tracheostomy tube-related additional work of breathing was highest in continuous positive airway pressure and was reduced most effectively in automatic tube compensation mode. ${ }^{28}$ An observational study of 24 patients found that the dead-space and airway resistance of tracheostomy tubes were comparable to endotracheal tubes, contrary to common perception. ${ }^{29}$

\section{Management of the cuff}

Eight studies addressed issues related to cuff inflation management. One was an anecdotal case series ${ }^{30}$ and another was an abstract aimed at optimising adherence to cuff inflation pressures between $20 \mathrm{~mm} \mathrm{Hg}$ and 30 mm Hg. ${ }^{31}$ Three studies investigated the impact of cuff deflation on respiratory parameters during positive pressure ventilation or weaning. ${ }^{32-34}$ One measured airway pressure in 16 patients and found clinically insignificant decreases in end-expiratory pressure with cuff deflation; however, all patients could vocalise and three quarters $(n=12)$ could swallow effectively. ${ }^{32}$ In a report of a series of long-term wean patients, most (99/104; 95\%) were found to be managed safely with either deflated cuffs or cuffless tubes. ${ }^{33}$ Another investigation with T-piece trials among 13 patients found that diaphragmatic effort was significantly lower with cuff deflation as compared with inflation. ${ }^{34}$

One retrospective review of 113 patients found that $95 \%(n=107)$ of patients tolerated cuff deflation on first attempt and that clinical stability and aspirated above-cuff secretions $\leq 1 \mathrm{~mL} / \mathrm{h}$ were highly predictive of success. ${ }^{35}$ Another retrospective audit found that among 30 patients, use of an early cuff deflation strategy resulted in a decreased ICU length of stay (28 vs 45 days) and mean number of tracheostomy days (16 vs 38) compared with historical controls. ${ }^{36}$ Hernandez et al conducted a clinical trial comparing cuff deflation and inflation during spontaneous breathing trials. ${ }^{37}$ They found that cuff deflation resulted in a significantly shorter wean time (3 vs 8 days, $\mathrm{p}<0.01)$ with an associated significantly lower incidence of respiratory infection ( $20 \mathrm{vs} 36 \%, \mathrm{p}=0.02$ ).

\section{Tracheostomy change and revision}

Two studies investigated tracheostomy changes or revision. In an observational study of 130 stable respiratory care unit patients, tracheostomy changes before 7 days were associated with earlier liberation from the ventilator (2.2 vs 3.9 days), use of speaking valves (7 vs 12 days), oral intake (10 vs 20 days) and discharge from the ICU (11 vs 17 days).$^{38}$ It may be argued that this population is more likely to have a better prognosis and that outcomes may not be directly related to early tracheostomy change. A survey of Dutch ICUs found that $59 \%$ of units $(n=26)$ did not routinely change the tracheostomy. ${ }^{39}$ One phenomenological study explored the patient experience of tracheostomy changes, and identified four key themes including physical sensation, psychological preparation, essentialness of communication and trust and competence. $^{40}$

\section{Optimisation of speech and communication}

Twenty-eight articles investigated the optimisation of speech and communication. One-way speaking valves are 
widely used and were found to safely achieve successful phonation in most patients who were fit for cuff deflation, with adverse effects rare. ${ }^{41}$ A 1993 prospective study found that one-way speaking valves can be used to facilitate communication during positive pressure ventilation; however, this method has largely been superseded by newer adjuncts. ${ }^{42}$

Studies suggest that in-line speaking valves can significantly hasten time to phonation in patients who are unable to tolerate cuff deflation. ${ }^{4-45}$ A randomised clinical trial of 30 ventilated patients found that, compared with cuff deflation with one-way speaking valves, early speech interventions with in-line speaking valves reduced time to phonation ( 7 vs 18 days, $\mathrm{p}=0.001$ ), with no increase in time to decannulation, time of ventilation, length of stay or adverse events. ${ }^{44}$ Further, Sutt et al used electrical impedance tomography to demonstrate that in-line speaking valves can improve gas distribution and alveolar recruitment. ${ }^{46}$

There were mixed findings on the role of fenestrated tubes; however, both studies that considered this topic were low-quality evidence studies including one abstract. ${ }^{47} 48$

Several studies evaluated tracheostomy 'talk tubes'. ${ }^{49-53}$ In those studies, $90 \%-100 \%$ of patients were able to achieve phonation, with $78 \%-100 \%$ adequately intelligible for effective communication. ${ }^{49-53}$ Excessive secretions and cough were the most common adverse effects. ${ }^{4-51}$ There was no demonstrated significant air leak when using the devices, even at higher pressures, and no mucosal injuries on bronchoscopy. ${ }^{50}$ An interventional study of 20 patients found that patients using Portex 'Talk' tubes had shorter time to phonation compared with Communi-Trach I tubes (2.1 vs 5.6 days, $\mathrm{p}<0.001){ }^{52}$

A feasibility study of above-cuff vocalisation involving 10 patients found that this technique resulted in phonation in eight patients, with $72 \%$ (66/91) of attempts resulting in audible speech. ${ }^{45}$

Three articles addressed the use of augmentative and alternative communication devices, with all finding statistically significant improvements in a patient's ability to communicate. One prospective pilot study involving 12 patients found $50 \%(\mathrm{n}=6)$ reported frustration with the use of these devices. ${ }^{54}$ Two studies also included communication skills training of doctors and nurses caring for patients uses the devices, and found this resulted in significant improvements in their ability to communicate with patients. ${ }^{556}$

Eight studies evaluated patient experience or quality of life relating to speech or communication. The participants highlighted that feelings of powerlessness, being misunderstood and physical discomfort impacted on their quality of life. Patients also acknowledged the importance of non-verbal communication. ${ }^{57-59}$ One study interviewed both patients and nurses and identified mutual experiences of frustration and powerlessness attributable to communication difficulties. ${ }^{59}$ One randomised controlled trial used talk tubes and found significant improvements in voice-related quality of life..$^{53}$ Another used in-line speaking valves and favoured the use of in-line valves over one-way speaking valve but found that the improvement in quality of life was not statistically significant. ${ }^{44}$

\section{Optimisation of swallow}

Nine studies considered the optimisation of swallowing and oral intake. The incidence of swallowing dysfunction in tracheostomised patients has been reported as between $30 \%$ and $70 \%$; however, several factors can increase this risk, including pre-existing swallowing impairment, neurological disorders, age, sedation and hypercarbia. ${ }^{60-63}$ In a study excluding patients with neurological disorders, 38\% (15/40) had swallowing dysfunction, suggesting that previous studies may have overestimated incidence based on this patient group. ${ }^{62}$

Two Australian studies found almost $80 \%$ of tracheostomised patients were seen by speech-language pathologists. ${ }^{60}{ }^{64}$ While the majority $(86 \% ; 108 / 126)$ of patients will commence oral intake with a tracheostomy in situ, only $43 \%(54 / 126)$ of patients had commenced oral intake in the ICU. ${ }^{60} \mathrm{~A}$ prospective study of 14 patients evaluated a swallowing rehabilitation programme administered by speech-language pathologists and an otorhinolaryngologist. ${ }^{65}$ Ten of the 14 patients $(71 \%)$ were able to receive oral intake, with $6(42 \%)$ having complete resolution of dysphagia, and 2 (14\%) experiencing partial improvement.

There are multiple methods for assessing swallow in the tracheostomised patient. ${ }^{64}$ One abstract found the Evans Blue Dye Test had a sensitivity of $84 \%$ and specificity of $100 \%$ when compared with fibreoptic endoscopic evaluation of swallow. ${ }^{66}$ A prospective study involving 35 patients found 12 patients (34\%) had a swallowing abnormality on bedside assessment. Of those 12 patients, and a further 7 patients with a high index of suspicion, $83 \%$ had abnormal swallow on videofluoroscopy ${ }^{63}$

Three studies considered specific elements of swallow physiology, including the impact of cuff pressure and subglottic insufflation, finding that increased cuff pressure influences both the sensory and motor components of the swallow reflex. ${ }^{6768}$ A prospective cross-over randomised and physiological study of 16 patients investigated the impact of meals on respiratory mechanics. ${ }^{69}$ While there was a significant increase in respiratory rate, tidal volume and subjective dyspnoea, this was not associated with desaturation or cardiovascular instability. ${ }^{69}$

\section{Other}

Several articles were included that did not directly fit within the a priori themes according to the research questions posed.

Three articles addressed stomal care and complications, including the dressing choice, wound granulation and pressure injuries. ${ }^{70-72}$ The most common 
complications relating to stoma care in the acute phase are bleeding, infection and granulation; however, their incidence varies between sources from $4 \%$ to $40 \%$. $^{70} 7374$ In an academic poster that assessed 23 possible risk factors for pressure injuries, percutaneous tracheostomy was the only factor that reached statistical significance in univariate analysis. ${ }^{71} \mathrm{~A}$ randomised trial found no difference in the incidence of stomal infection when using gauze dressings compared with absorbent foam $(17.5 \% \mathrm{v} 10 \%$, $\mathrm{p}=0.051){ }^{70}$

Nine articles addressed the topic of tracheostomy bacterial colonisation, and tracheostomy-associated and ventilator-associated pneumonia. Oral care bundles with surveillance and auditing protocols were found to significantly reduce the incidence of tracheostomy-associated or ventilator-associated pneumonia. ${ }^{75}$ Studies also found that suction-above-the-cuff in tracheostomised patients reduced infection and microbial quantity. ${ }^{77-79}$ A randomised controlled trial (abstract) of 19 patients found the use of speaking valves during weaning reduced the incidence of nosocomial respiratory infection and may reduce time to decannulation. ${ }^{80}$

One cohort study (abstract) found that $89 \%(67 / 75)$ of tracheostomised patients in ICU reported trouble falling asleep. ${ }^{81}$ Studies found that sleep time and efficiency were poor, and that sleep time was not improved by melatonin ( 240 vs $243 \mathrm{~min}, \mathrm{p}=0.68$ for melatonin vs placebo). ${ }^{82} 83$ Patients ventilated with mechanical ventilation compared with spontaneously ventilating had a significantly longer total sleep time ( 183 vs $132 \mathrm{~min}, \mathrm{p}=0.04$ ); however, there was no improvement in rapid eye movement sleep or sleep fragmentation. ${ }^{83}$ An observational study found that after insertion of a tracheostomy, there was a significant reduction in sedative drug requirements and time spent 'heavily sedated' which was reduced from 7 hours per day to one. ${ }^{84}$

Four studies (including three abstracts) evaluated the impact of multidisciplinary tracheostomy teams, finding they resulted in reduced time to first oral intake, first tube change and decannulation, and reduced length of stay and complication rates. ${ }^{85-88}$ Reduced time to weaning and decannulation were noted in three of the four studies. ${ }^{85} 8788$

Three studies including one abstract addressed quality of life or stressful experiences, with patients reporting that psychosocial discomfort, sleeplessness, physical symptoms of thirst and pain, fear and relationships had a significant impact on their experience. ${ }^{8189} 90$

Four studies (including three abstracts) were observational audits, ${ }^{91-94}$ three (including two abstracts) surveyed participants' knowledge on an aspect of tracheostomy care $^{95-97}$ and two were surveys regarding standards of practice. ${ }^{398}$ There was one clinical consensus statement published by the American Academy of OtolaryngologyHead and Neck Surgery Foundation, using a Delphi process. ${ }^{99}$

\section{DISCUSSION}

In this scoping review, we identified and explored five main themes related to the management of patients with a tracheostomy from time post insertion to ICU discharge. Despite slow-growing interest in this area, research remains limited. There were a significant number of low-quality studies and abstracts published only in conference proceedings without progression to peer-reviewed publication, and a lack of clinical trials.

Weaning from the ventilator is an integral part of practice in critical care medicine. In our review, we found that there is support for the use of protocols, including nurse-led protocols; that T-piece and high-flow oxygen delivery may be used during weaning to improve oxygenation; and that diaphragmatic strengthening may improve weaning success. ${ }^{21} 23-25$

Personal experience and anecdotal surveys of intensivists have revealed vast practice variation in cuff management both within and between countries. ${ }^{396064}$ Some clinicians view cuff deflation as a late step in the weaning process, while others view cuff deflation, tube downsizing and exchange to cuffless tubes, as the standard practice. The former view may, in part, be related to an erroneous belief that cuff inflation during weaning reduces aspiration and associated pneumonia. ${ }^{6061} 100$ The available evidence indicates that early cuff deflation is widely tolerated by patients during weaning, can be reliably predicted based on clinical assessment, facilitates speech and oral intake, reduces time to weaning and decannulation, and can reduce rates of respiratory infections. ${ }^{32-37}$ While data are limited, there is potential benefit from early tracheostomy downsizing or switching to cuffless tubes. ${ }^{3860} 88$

There is a significant body of literature that supports the optimisation of speech and communication as it is important from a rehabilitative and psychoemotional point of view. ${ }^{44} 53101$ Many of the strategies for enhancing communication that were considered in the included articles have been demonstrated as safe, simple to use and improving both communication and quality of life. ${ }^{43-46} 58102$ Speech adjuncts, used alongside strategies for early cuff deflation, may lead to earlier weaning, fewer complications and improved patient experience, and may also lead to earlier oral intake, with its associated benefits. ${ }^{434453}$

In the course of this review, we have identified several areas of clinical importance that have not been adequately explored in the literature. While not an exhaustive list, we believe the 10 topics listed in table 2 should be considered as priorities for further research.

There are some methodological aspects of our study that merit discussion. Our report benefits from the use of a structured, predefined scoping review methodology, as outlined by The Joanna Briggs Institute. ${ }^{12}$ As the objective was to map the evidence rather than conduct a critical appraisal, and as is recommended for the scoping review methodology, we made no further attempts to grade the quality of the included reports. ${ }^{12}{ }^{13}$ We recognised in advance of our study that there may be a small body of 
Table 2 Research priorities related to tracheostomy care in intensive care

1. Approaches to weaning, with comparisons between methods such as spontaneous breathing trials, reducing ventilatory support and high-flow oxygen.

2. The role of protocolised and nurse-led weaning.

3. The patient experience of weaning, and the impact of mood and motivation on outcomes.

4. The impact of early cuff deflation or cuffless tubes on weaning outcomes and optimisation of speech and oral intake.

5. The role and timing of tracheostomy tube changes (including exchanging for smaller, cuffless or fenestrated tubes), and the impact on stomal complications.

6. Comparative trials of the range of speaking adjuncts, with a focus on speech intelligibility, and benefits relating to alveolar recruitment.

7. Clinical trials of above-cuff vocalisation, with consideration of potential benefits, such as swallowing rehabilitation, and risks, such as aspiration.

8. Screening for readiness for oral intake trials and factors that contribute to delays in the commencement of oral intake.

9. The patient experience of oral intake, or lack thereof, and its impact on quality of life.

10. The role of tracheostomy multidisiciplinary teams in the intensive care setting.

literature and therefore used broad search terms with the resulting need to screen more than 6000 citations. However, we limited our search to the three most widely used citations databases and therefore may have missed articles not included within these. Finally, it should be recognised that we limited our scope of review to the stay in ICU. As such, there may be other management issues that could arise post-ICU discharge that are not included in our investigative themes.

\section{CONCLUSION}

In summary, this scoping review addresses an area of the critical care literature that, while of major clinical importance, has garnered relatively little attention. While major efforts have been expended to investigate the optimal decision-making for, and timing and insertion of, tracheostomies in critically ill patients, the body of knowledge surrounding management of tracheostomies in intensive care is limited by low-quality and non-peer reviewed research. While there has been an increase in research in this area (figure 2), it is evident that there needs to be a shift in our research focus from the conduct of the procedure itself to optimising the management of the tracheostomised patients within our ICUs.

Contributors KBL takes responsibility for the content of the manuscript including data and analysis, as the guarantor. KBL and KAW made substantial contribution to the conception and design of the work. All authors were involved in the acquisition, analysis and interpretation of data. All authors were involved in drafting and revising the manuscript and approved the version to be published. All authors agreed to be accountable for all aspects of the work in ensuring that questions relating to the accuracy or integrity of any part of the work are appropriately investigated and resolved.

Funding The authors have not declared a specific grant for this research from any funding agency in the public, commercial or not-for-profit sectors.

Competing interests None declared.

Patient consent for publication Not required.

Provenance and peer review Not commissioned; externally peer reviewed.
Data availability statement All data relevant to the study are included in the article or uploaded as supplementary information.

Open access This is an open access article distributed in accordance with the Creative Commons Attribution Non Commercial (CC BY-NC 4.0) license, which permits others to distribute, remix, adapt, build upon this work non-commercially, and license their derivative works on different terms, provided the original work is properly cited, appropriate credit is given, any changes made indicated, and the use is non-commercial. See: http://creativecommons.org/licenses/by-nc/4.0/.

ORCID IDs

Kirsty A Whitmore http://orcid.org/0000-0003-2787-0789

Kevin B Laupland http://orcid.org/0000-0002-1205-5354

\section{REFERENCES}

1 Abe T, Madotto F, Pham T, et al. Epidemiology and patterns of tracheostomy practice in patients with acute respiratory distress syndrome in ICUs across 50 countries. Crit Care 2018;22:195.

2 Klotz R, Probst P, Deininger M, et al. Percutaneous versus surgical strategy for tracheostomy: a systematic review and meta-analysis of perioperative and postoperative complications. Langenbecks Arch Surg 2018;403:137-49.

3 Freeman BD. Tracheostomy update: when and how. Crit Care Clin 2017:33:311-22.

4 Herritt B, Chaudhuri D, Thavorn K, et al. Early vs. late tracheostomy in intensive care settings: impact on ICU and hospital costs. $J$ Crit Care 2018:44:285-8.

5 Perkins MP, King C, Moores LK. Early versus late tracheostomy: a metaanalysis. Chest2009;136:16S.

6 Jerjes W, Hamdoon Z, Abbas S, et al. Percutaneous dilational tracheostomy and open surgical tracheostomy: systematic review. Brit J Oral Max Surg 2011;49:S69.

7 Siempos II, Ntaidou TK, Filippidis FT, et al. Effect of early versus late or no tracheostomy on mortality and pneumonia of critically ill patients receiving mechanical ventilation: a systematic review and meta-analysis. Lancet Respir Med 2015;3:150-8.

8 Pan L, Guo Y. Effect of early versus late tracheotomy in critically ill patients: a systematic review and meta-analysis. Chest 2016;149:A165.

9 Gobatto ALN, Besen BAMP, Cestari M, et al. Ultrasound-Guided percutaneous dilational tracheostomy: a systematic review of randomized controlled trials and meta-analysis. J Intensive Care Med 2020;35:445-52.

10 Stelfox H, Crimi C, Berra L, et al. Determinants of tracheostomy decannulation: an international survey. Crit Care 2008;12:R26.

11 Zanata IdeL, Santos RS, Hirata GC. Tracheal decannulation protocol in patients affected by traumatic brain injury. Int Arch Otorhinolaryngol 2014;18:108-14. 
12 Peters MDJ, Godfrey CM, Khalil H, et al. Guidance for conducting systematic scoping reviews. Int J Evid Based Healthc 2015;13:141-6.

13 Munn Z, Peters MDJ, Stern C, et al. Systematic review or scoping review? Guidance for authors when choosing between a systematic or scoping review approach. BMC Med Res Methodol 2018;18:143.

14 Fagoni N, Piva S, Peli E, et al. Comparison between a nurseled weaning protocol and weaning based on physician's clinical judgment in tracheostomized critically ill patients: a pilot randomized controlled clinical trial. Ann Intensive Care 2018;8:11.

15 Ceriana P, Carlucci A, Navalesi $P$, et al. Weaning from tracheotomy in long-term mechanically ventilated patients: feasibility of a decisional flowchart and clinical outcome. Intensive Care Med 2003;29:845-8

16 Hoffman LA, Miller TH, Zullo TG, et al. Comparison of 2 models for managing tracheotomized patients in a subacute medical intensive care unit. Respir Care 2006;51:1230-6.

17 van der Lely A-JW, Veelo DP, Dongelmans DA, et al. Time to wean after tracheotomy differs among subgroups of critically ill patients: retrospective analysis in a mixed medical/surgical intensive care unit. Respir Care 2006;51:1408-15.

18 Pattani H, Ehlers M, Girling K, et al. Pilot study of two nurse-led weaning protocols in patients with tracheostomies. $J$ Intensive Care Soc 2014:15:18-23.

19 Bach JR, Saporito LR. Criteria for extubation and tracheostomy tube removal for patients with ventilatory failure. A different approach to weaning. Chest 1996;110:1566-71.

20 Carlucci A, Ceriana P, Prinianakis G, et al. Determinants of weaning success in patients with prolonged mechanical ventilation. Crit Care 2009;13:R97.

21 Lovas A, Molnár Z. T-Piece improves arterial and central venous oxygenation in trachestomized patients as compared to continuous positive airway pressure/pressure support ventilation. Minerva Anestesiol 2013;79:492-7.

22 Michalopoulos AS, Gregoriades K, Falagas ME. The effect of different arrangements of T-piece parts on oxygenation of patients with tracheostomy. Anesth Analg 2006;103:1054-5.

23 Corley A, Edwards M, Spooner AJ, et al. High-Flow oxygen via tracheostomy improves oxygenation in patients weaning from mechanical ventilation: a randomised crossover study. Intensive Care Med 2017;43:465-7

24 Mitaka C, Odoh M, Satoh D, et al. High-Flow oxygen via tracheostomy facilitates weaning from prolonged mechanical ventilation in patients with restrictive pulmonary dysfunction: two case reports. J Med Case Rep 2018;12.

25 Natalini D, Idone FA, Grieco DL, et al. Impact of high-flow oxygen therapy delivered through a tracheostomy on arterial blood gases and endotracheal pressure. Crit Care 2014;18:P321.

26 Gonzalez I, Jimenez P, Valdivia J, et al. Effectiveness of humidification with heat and moisture exchanger-booster in tracheostomized patients. Indian J Crit Care Med 2017;21:528-30.

27 Thomachot L, Viviand X, Arnaud S, et al. Preservation of humidity and heat of respiratory gases in spontaneously breathing, tracheostomized patients. Acta Anaesthesiol Scand 1998;42:841-4.

28 Haberthür C, Fabry B, Stocker R, et al. Additional inspiratory work of breathing imposed by tracheostomy tubes and non-ideal ventilator properties in critically ill patients. Intensive Care Med 1999;25:514-9

29 Joseph MJ, Khoury A, Mendoza AE, et al. Tracheostomy in the critically ill: the myth of dead space. Anaesth Intensive Care 2013;41:216-21.

30 Higgins DM, Maclean JC. Dysphagia in the patient with a tracheostomy: six cases of inappropriate cuff deflation or removal. Heart Lung 1997;26:215-20.

31 Allam M, Augustyn C, Ramsay G, et al. An audit of endotracheal tube and tracheostomy cuff pressure monitoring in a surgical intensive care unit. Southern African Journal of Critical Care 2019;35:33

32 Conway $\mathrm{DH}$, Mackie $\mathrm{C}$. The effects of tracheostomy cuff deflation during continuous positive airway pressure*. Anaesthesia 2004;59:652-7.

33 Bach JR, Alba AS. Tracheostomy ventilation. A study of efficacy with deflated cuffs and cuffless tubes. Chest 1990;97:679-83.

34 Ceriana P, Carlucci A, Navalesi P, et al. Physiological responses during a T-piece weaning trial with a deflated tube. Intensive Care Med 2006;32:1399-403.

35 Pryor LN, Ward EC, Cornwell PL, et al. Clinical indicators associated with successful tracheostomy cuff deflation. Aust Crit Care 2016;29:132-7.
36 Callon J, Lamont C, Dyson S, et al. Early cuff deflation in tracheostomised patients requiring ventilatory support. Critical Care 2019;23.

37 Hernandez G, Pedrosa A, Ortiz R, et al. The effects of increasing effective airway diameter on weaning from mechanical ventilation in tracheostomized patients: a randomized controlled trial. Intensive Care Med 2013;39:1063-70.

38 Fisher DF, Kondili D, Williams J, et al. Tracheostomy tube change before day 7 is associated with earlier use of speaking valve and earlier oral intake. Respir Care 2013;58:257-63.

39 Veelo DP, Schultz MJ, Phoa KYN, et al. Management of tracheostomy: a survey of Dutch intensive care units. Respir Care 2008;53:1709-15.

40 Donnelly $F$, Wiechula $R$. The lived experience of a tracheostomy tube change: a phenomenological study. J Clin Nurs 2006;15:1115-22.

41 Fröhlich MR, Boksberger H, Barfuss-Schneider C, et al. Safe swallowing and communicating for ventilated intensive care patients with tracheostoma: implementation of the Passy Muir speaking valve. Pflege 2017;30:387-94.

42 Manzano JL, Lubillo S, Henriquez D, et al. Verbal communication of ventilator-dependent patients. Crit Care Med 1993;21:512-7.

43 Sutt A-L, Cornwell P, Mullany D, et al. The use of tracheostomy speaking valves in mechanically ventilated patients results in improved communication and does not prolong ventilation time in cardiothoracic intensive care unit patients. J Crit Care 2015;30:491-4.

44 Freeman-Sanderson AL, Togher L, Elkins MR, et al. Return of voice for ventilated tracheostomy patients in ICU: a randomized controlled trial of Early-Targeted intervention. Crit Care Med 2016;44:1075-81.

45 McGrath BA, Wallace S, Wilson M, et al. Safety and feasibility of above cuff vocalisation for ventilator-dependant patients with tracheostomies. J Intensive Care Soc 2019;20:59-65.

46 Sutt A-L, Anstey CM, Caruana LR, et al. Ventilation distribution and lung recruitment with speaking valve use in tracheostomised patient weaning from mechanical ventilation in intensive care. $J$ Crit Care 2017;40:164-70.

47 Maistry N, De Sousa P. Do fenestrated tracheostomy tubes still have a place in ICU? outcomes from an audit conducted in two intensive care units at a specialised tertiary centre. J Intensive Care Soc 2019;20:45.

48 Fukumoto $\mathrm{M}$, Ota $\mathrm{H}$, Arima $\mathrm{H}$. Ventilator weaning using a fenestrated tracheostomy tube with a speaking valve. Crit Care Resusc 2006;8:117-9.

49 Kunduk M, Appel K, Tunc M, et al. Preliminary report of laryngeal phonation during mechanical ventilation via a new cuffed tracheostomy tube. Respir Care 2010;55:1661-70.

50 Nomori $\mathrm{H}$. Tracheostomy tube enabling speech during mechanical ventilation. Chest 2004;125:1046-51.

51 Sparker ANNW, Robbins KT, Nevlud GN, et al. A prospective evaluation of speaking tracheostomy tubes for ventilator dependent patients. Laryngoscope 1987:97:89-92.

52 Leder SB. Verbal communication for the ventilator-dependent patient: Voice intensity with the portex "talk" $₫$ tracheostomy tube. Laryngoscope 1990;100:1116-21.

53 Pandian V, Cole T, Kilonsky D, et al. Voice-Related quality of life increases with a talking tracheostomy tube: a randomized controlled trial. Laryngoscope 2020;130:1249-55.

54 Garry J, Casey K, Cole TK, et al. A pilot study of eye-tracking devices in intensive care. Surgery 2016;159:938-44.

55 Maringelli F, Brienza N, Scorrano F, et al. Gaze-controlled, computer-assisted communication in Intensive Care Unit: "speaking through the eyes". Minerva Anestesiol 2013;79:165-75

56 Happ MB, Garrett KL, Tate JA, et al. Effect of a multi-level intervention on nurse-patient communication in the intensive care unit: results of the SPEACS trial. Heart Lung 2014;43:89-98.

57 Flinterud SI, Andershed B. Transitions in the communication experiences of tracheostomised patients in intensive care: a qualitative descriptive study. J Clin Nurs 2015;24:2295-304.

58 Freeman-Sanderson AL, Togher L, Elkins M, et al. Quality of life improves for tracheostomy patients with return of voice: a mixed methods evaluation of the patient experience across the care continuum. Intensive Crit Care Nurs 2018;46:10-16.

59 Tolotti A, Bagnasco A, Catania G, et al. The communication experience of tracheostomy patients with nurses in the intensive care unit: a phenomenological study. Intensive Crit Care Nurs 2018;46:24-31.

60 Pryor L, Ward E, Cornwell P, et al. Patterns of return to oral intake and decannulation post-tracheostomy across clinical populations 
in an acute inpatient setting. Int $J$ Lang Commun Disord 2016;51:556-67.

61 Elpern EH, Scott MG, Petro L, et al. Pulmonary aspiration in mechanically ventilated patients with tracheostomies. Chest 1994;105:563-6.

62 Romero CM, Marambio A, Larrondo J, et al. Swallowing dysfunction in nonneurologic critically ill patients who require percutaneous dilatational tracheostomy. Chest 2010;137:1278-82.

63 Tolep K, Leonard Getch C, Criner GJ. Swallowing dysfunction in patients receiving prolonged mechanical ventilation. Chest 1996;109:167-72.

64 Freeman-Sanderson A, Togher L, Phipps P, et al. A clinical audit of the management of patients with a tracheostomy in an Australian tertiary hospital intensive care unit: focus on speech-language pathology. Int J Speech Lang Pathol 2011;13:518-25.

65 Rodrigues KA, Machado FR, Chiari BM, et al. Swallowing rehabilitation of dysphagic tracheostomized patients under mechanical ventilation in intensive care units: a feasibility study. Rev Bras Ter Intensiva 2015;27:64-71.

66 Garach MM, Romero OM, Puerta RR, et al. Accurancy of extended Evan's blue dye testing in predicting artificial airway dysphagia. Intensive Care Med Exp 2016;4.

67 Amathieu R, Sauvat S, Reynaud P, et al. Influence of the cuff pressure on the swallowing reflex in tracheostomized intensive care unit patients. Br J Anaesth 2012;109:578-83.

68 Clarett M, Andreu MF, Salvati IG, et al. [Effect of subglottic air insufflation on subglottic pressure during swallowing]. Med Intensiva 2014:38:133-9.

69 Vitacca M, Callegari G, Sarvà M, et al. Physiological effects of meals in difficult-to-wean tracheostomised patients with chronic obstructive pulmonary disease. Intensive Care Med 2005;31:236-42.

70 Ahmadinegad M, Lashkarizadeh MR, Ghahreman M, et al. Efficacy of dressing with absorbent foam versus dressing with gauze in prevention of tracheostomy site infection. Tanaffos 2014;13:13-19.

71 Hwang HS, Luk LJ, Kezirian EJ, et al. Tracheotomy-related pressure sores in critically ill patients. Laryngoscope 2011;121:S249.

72 Leder SB, Astrachan DI. Stomal complications and airflow line problems of the Communi-Trach I?? cuffed talking tracheotomy tube. Laryngoscope 1989;99:194-6.

73 Engels PT, Bagshaw SM, Meier M, et al. Tracheostomy: from insertion to decannulation. Can J Surg 2009;52:427-33.

74 Kumar VA, Reddy BU, Kiran Kumar VA, et al. Speech and swallowing function outcome following early tracheostomy in patients who underwent neurosurgical intervention. Indian J Crit Care Med 2018;22:427-30.

75 Eid RC, Domingues F, Silva Barreto JK, et al. Successful prevention of tracheostomy associated pneumonia in step-down units. $A m \mathrm{~J}$ Infect Control 2011;39:500-5.

76 Conley P, McKinsey D, Graff J, et al. Does an oral care protocol reduce VAP in patients with a tracheostomy? Nursing 2013;43:18-23.

77 Ledgerwood LG, Salgado MD, Black H, et al. Tracheotomy tubes with suction above the cuff reduce the rate of ventilator-associated pneumonia in intensive care unit patients. Ann Otol Rhinol Laryngol 2013;122:3-8.

78 Rabach L, Siegel MD, Puchalski JT, et al. Use of the Blom tracheotomy tube with suction inner cannula to Decontaminate microorganisms from the subglottic space. A proof of concept. Ann Am Thorac Soc 2015;12:859-63.

79 Terragni P, Brazzi L, Falco D, et al. Occurrence of ventilator associated pneumonia using tracheostomy tubes with subglottic secretion drainage. Critical Care 2017;21

80 Fernández Carmona A, Arias Díaz M, Aguilar Alonso E, et al. Use of speaking valve on preventing respiratory infections, in critical TRAQUEOSTOMIZED patients diagnosed of dysphagia secondary to artificial airway. EDISVAL study. Intensive Care Med Exp 2015;3:A936.
81 Riera MA, Gallart E, Afonso E, et al. Stressful perceptions of tracheostomized patients in the intensive care unit. Intensive Care Med 2014:40:S275.

82 Ibrahim MG, Bellomo R, Hart GK, et al. A double-blind placebocontrolled randomised pilot study of nocturnal melatonin in tracheostomised patients. Crit Care Resusc 2006;8:187-91.

83 Roche-Campo F, Thille AW, Drouot X, et al. Comparison of sleep quality with mechanical versus spontaneous ventilation during weaning of critically ill Tracheostomized Patients*. Crit Care Med 2013;41:1637-44.

84 Nieszkowska A, Combes A, Luyt C-E, et al. Impact of tracheotomy on sedative administration, sedation level, and comfort of mechanically ventilated intensive care unit patients*. Crit Care Med 2005;33:2527-33

85 Al Sindi M, Sarwani SN, Sarwani O. The impact of specialized tracheostomy care team. Bahrain Medical Bulletin 2016;38:94-6.

86 Bocci M, Todd SR, Hargett K, et al. A team approach to mechanically ventilated patients with tracheostomy in a surgical ICU reduces length of stay and mortality. Critical Care Med 2009;37:A440.

87 Santos A, Harper D, Gandy S, et al. 1214. Crit Care Med 2018;46:591.

88 Welton C, Morrison M, Catalig M, et al. Can an interprofessional tracheostomy team improve weaning to decannulation times? A quality improvement evaluation. Can J Respir Ther 2016;52:7-11.

89 Pandian V, Bose S, Miller C, et al. Exploring quality of life in critically ill tracheostomy patients: a pilot study. ORL Head Neck Nurs 2014;32:6-8. 10-3.

90 Foster A. More than nothing: the lived experience of tracheostomy while acutely ill. Intensive Crit Care Nurs 2010;26:33-43.

91 Davies MG, Horton D, Chadwick R, et al. S135 Long-term outcomes in patients referred to a specialised weaning centre; The impact of referral source, non-invasive ventilation and diagnosis. Thorax 2013;68:A69.2-70.

92 Elise M, Julie D, Martin D, et al. Prolonged ICU stays and difficultto-wean-patients: first year of experience in a French post ICU rehabilitation center. Annals of Intensive Care 2018;8.

93 Haniez F, Jaillet $\mathrm{H}$, Maas $\mathrm{H}$, et al. First-Year experience of a French mechanical ventilation weaning centre. Annals of Intensive Care 2017;7:53-4.

94 Marchese S, Corrado A, Scala R, et al. Tracheostomy in patients with long-term mechanical ventilation: a survey. Respir Med 2010;104:749-53

95 Cumpstey A, Horwell E, Benham S, et al. Teaching Foundation doctors about tracheostomy complications: a 6 month follow up study. Intensive Care Medicine 2013;39:S253.

96 Mol DA, De Villiers Gdu T, Claassen AJ, et al. Use and care of an endotracheal/tracheostomy tube cuff-are intensive care unit staff adequately informed? S Afr J Surg 2004;42:14-16.

97 Orchard W, Siviter R. Management of displaced tracheostomies in the intensive care unit. Anaesthesia 2018;73:65.

98 Blondonnet R, Chabanne R, Godet T, et al. [Tracheostomy in French ICUs and patient outcome: national opinion survey]. Ann Fr Anesth Reanim 2014;33:227-31.

99 Mitchell RB, Hussey HM, Setzen G, et al. Clinical consensus statement: tracheostomy care. Otolaryngol Head Neck Surg 2013;148:6-20.

100 Young PJ, Burchett K, Harvey I, et al. The prevention of pulmonary aspiration with control of tracheal wall pressure using a silicone cuff. Anaesth Intensive Care 2000;28:660-5.

101 Ten Hoorn S, Elbers PW, Girbes AR, et al. Communicating with conscious and mechanically ventilated critically ill patients: a systematic review. Crit Care 2016;20:333.

102 Freeman-Sanderson AL, Togher L, Elkins MR, et al. Quality of life improves with return of voice in tracheostomy patients in intensive care: an observational study. J Crit Care 2016;33:186-91. 